\title{
Transcatheter aortic valve implantation complicated by acute pericardial tamponade
}

\author{
Olga Możeńska¹, Piotr Suwalski², Agnieszka Pawlak², Tomasz Kulawik², Katarzyna Byczkowska², Robert J. Gil² \\ 1Department of Noninvasive Cardiology and Hypertension, Central Clinical Hospital of the Ministry of Interior, Warsaw, Poland \\ ${ }^{2}$ Department of Cardiac Surgery, Central Clinical Hospital of the Ministry of Interior, Warsaw, Poland
}

Postep Kardiol Inter 2014; 10, 1 (35): 26-28

DOI: 10.5114/pwki.2014.41463

\begin{abstract}
A bstract
Transcatheter aortic valve replacement (TAVR) is now an accepted standard of care for patients with symptomatic severe aortic stenosis who are not candidates for surgery or have high surgical risk. Despite its more widespread adoption as a treatment option and increasing experience of centers, TAVR is still associated with several complications. We therefore report a case of TAVR complicated by acute pericardial tamponade, one of the most severe potential complications of this procedure. We describe the way we approached the problem and we try to give a potential take-home message for others who might encounter such a situation in their own cath lab.
\end{abstract}

Key words: transcatheter aortic valve replacement, acute pericardial tamponade, aortic stenosis.

\section{Introduction}

Transcatheter aortic valve replacement (TAVR) is considered less invasive than conventional surgical aortic valve replacement because it avoids sternotomy and cardiopulmonary bypass. Despite increasing experience with TAVR, it can be associated with complications, which can be technically challenging, even for an experienced operator. Complications include vascular complications, valve malpositioning, regurgitation, embolization, coronary compromise, conduction abnormalities, stroke/transient ischemic attack, acute kidney injury, cardiac tamponade and hemodynamic collapse.

To our knowledge, there is no reported case of TAVR using Core Valve ${ }^{\mathrm{TM}}$ complicated by acute tamponade and followed by successful surgical repair, successful percutaneous prosthesis implantation and full patient recovery.

\section{Case report}

A 78-year-old obese female with coronary artery disease, hypertension, hyperlipidemia, and diabetes mellitus type 2 underwent TAVR due to severe aortic valve (AV) stenosis (Figure $1 \mathrm{~A}$ ). She presented clinically with NYHA class III, and CCS class III/IV. The patient was excluded from surgical AV repair due to concomitant diseases and the presence of a large abdominal hernia threatening with necrosis in case of cardiac pulmonary bypass use. The patient's EuroSCORE was 9.36\%, logistic EuroSCORE was $12.69 \%$ and STS for isolated AV replacement showed a mortality risk of $22.883 \%$. On transthoracic echocardiography AV parameters were: AVA $0.7 \mathrm{~cm}^{2}$, peak gradient $82 \mathrm{~mm} \mathrm{Hg}$, mean gradient $54 \mathrm{~mm} \mathrm{Hg}$ and $\mathrm{EF}$ 60\%. Transcatheter aortic valve replacement was performed through a transfemoral approach. During the procedure, while the operator was manipulating with the Amplatz Extrastiff wire, immediately after balloon predilatation, the patient's blood pressure dropped, bradycardia appeared and the patient went into cardiac arrest. Transesophageal echocardiography (TEE) showed features of acute cardiac tamponade (Figure $1 \mathrm{~B}$ ). Aortic ring rupture or aortic root rupture was suspected. Immediate sternotomy was performed and perforation of the left ventricle (LV) lateral wall was indentified and repaired with mattress sutures with pledgets. After achieving full hemostasis a decision about further proceeding with TAVR was made. A Core Valve ${ }^{\mathrm{TM}} 26 \mathrm{~mm}$ was successfully implanted (Figure $1 \mathrm{C}$ and $1 \mathrm{D})$.

\section{Corresponding author:}

Olga Możeńska MD, Department of Noninvasive Cardiology and Hypertension, Central Clinical Hospital of the Ministry of Interior, 137 Wołoska St, 02-507 Warsaw, Poland, phone: +48 609732 044, e-mail: ola_85@poczta.fm

Received: 21.10.2013, accepted: 9.01.2014. 

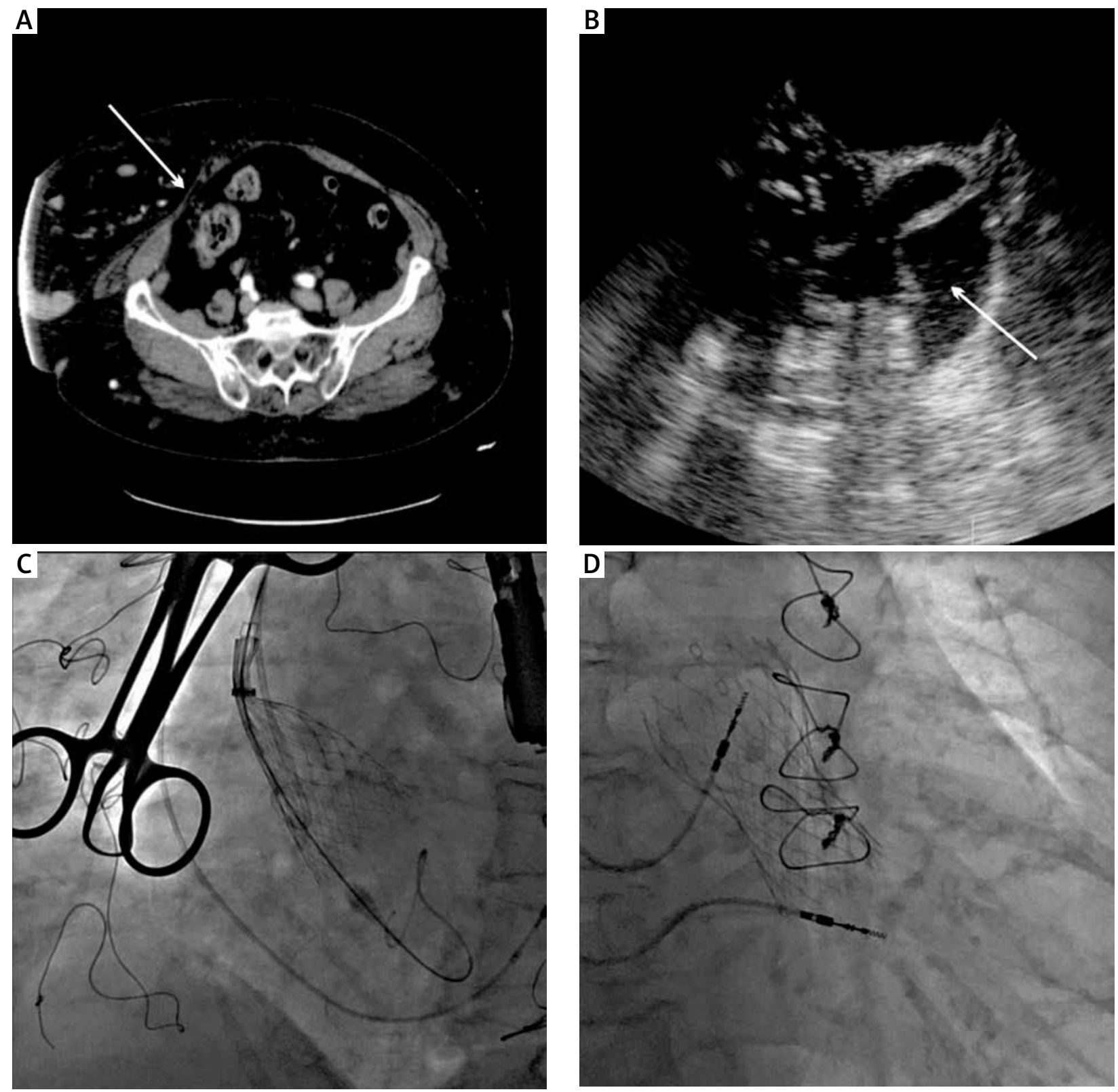

Figure 1. A - Severe abdominal hernia on abdominal computed tomography (CT). B - Acute cardiac tamponade shown with transesophageal echocardiography (TEE). C, D - Successful implantation of Core Valve ${ }^{\mathrm{TM}} 26 \mathrm{~mm}$

Acute pericardial tamponade seemed to have been the effect of apparently too aggressive wire manipulation and then of sudden contraction of the LV lateral wall as an answer to inotropes given by the anesthesiologist when the patient started crashing.

The patient definitely benefitted from TAVR, although the intraoperational decision making process was incredibly challenging. Transesophageal echocardiography continuous monitoring became a key to successful diagnosis of the cause of cardiac arrest and the participation of an experienced cardiothoracic surgeon was crucial. However, our main take-home message is to always remove the Amplatz Extrastiff wire from the LV if any need for inotropes during TAVR appears. As additional information we have over a year follow-up of the patient and her cardiac function. Currently her heart shows no signs of post-surgical repair damage. Her new valve function is correct with only mild regurgitation, peak gradient is $8 \mathrm{~mm} \mathrm{Hg}$, ejection fraction (EF) is $60 \%$ with no contractility abnormalities. Clinically she presents with NYHA class I/II and CCS class I. Additionally she has lost over $25 \mathrm{~kg}$ of weight.

\section{Discussion}

Transcatheter aortic valve replacement has emerged as a promising alternative to conventional aortic valve 
replacement for patients with severe, symptomatic aortic stenosis who are otherwise left untreated due to the perceived high risk of operative mortality [1]. Since the firstin-man procedure in 2002 [2] several improvements have been achieved in TAVR device technologies and procedural management, leading to incremental success rates [3-8]. With over 50,000 implants performed in more than 40 countries, a large amount of clinical data have emerged in this rapidly growing field. Careful patient selection, systematic risk stratification, optimal valve sizing, meticulous procedural techniques, and complication management are all important elements to achieve good outcomes.

Despite all that, complication rates are still quite high. Complications include stroke, myocardial infarction, bleeding, vascular injury such as perforation, dissection, trauma and arterial intussusception, device embolization, reverse placement of the stent valve, and geographic misplacement of the stent valve leading to the possible blocking of coronary ostia. Most of these complications can potentially be life threatening. Long-term complications include stroke, bleeding, paravalvular regurgitation, and endocarditis, although there have been case reports of a broad spectrum of rare complications that can occur.

In order to create a common language by which to quantify complications in a standardized and objective fashion, the Valve Academic Research Consortium (VARC) was formed. According to VARC criteria cardiac tamponade falls into the all-cause mortality primary end-point and further into the cardiovascular mortality secondary end-point. It also falls into prosthetic valve 'associated' complications and further into other prosthesis-related adverse events. Such an infrequent complication, according to VARC, includes new ventricular septal defects and aortic root rupture/perforation/dissection, occurring either during pre-implant balloon aortic valvuloplasty (as happened in our case), or during transcatheter valve implantation.

So, once we were caught in a very dynamic situation in the cath lab, during what we thought to be a rather straightforward TAVR case, we had a difficult time as a team. The first challenge was to make the correct diagnosis of what was happening with our patient. Once we decided that acute pericardial tamponade was responsible, the second question was how to repair it, knowing our patient's preprocedural risk scores. A fast decision about surgical repair was made, mainly due to aortic ring rupture or aortic root rupture being the main suspect of what was going on. After rather straightforward repair of perforation of the LV lateral wall the patient's status stabilized and our third biggest question arose: whether to repair the aortic stenosis, and if yes, then with what technique. Taking into consideration all the risks and benefits, the team decided to continue with the TAVR procedure. From then on it went smoothly with a very satisfactory final result.
Once the patient was safe in the intensive care unit, a discussion on potential causes and on whether our course of action was correct started. We came to the conclusion that acute pericardial tamponade was the effect of apparently too aggressive wire manipulation and then of sudden contraction of the LV lateral wall as a response to inotropes given by the anesthesiologist when the patient started crashing.

Our take-home message for other teams is to always remove the Amplatz Extrastiff wire from the LV if any need for inotropes during the TAVR procedure arises.

\section{References}

1. Zajarias A, Cribier AG. Outcomes and safety of percutaneous aortic valve replacement. J Am Coll Cardiol 2009; 53: 1829-1836.

2. Cribier A, Eltchaninoff $\mathrm{H}$, Bash $\mathrm{A}$, et al. Percutaneous transcatheter implantation of an aortic valve prosthesis for calcific aortic stenosis: first human case description. Circulation 2000; 106 : 3006-3008.

3. Cribier A, Eltchaninoff $\mathrm{H}$, Tron $\mathrm{C}$, et al. Treatment of calcific aortic stenosis with the percutaneous heart valve: mid-term follow-up from the initial feasibility studies: the French experience. J Am Coll Cardiol 2006; 47: 1214-1223.

4. Grube E, Schuler G, Buellesfeld L, et al. Percutaneous aortic valve replacement for severe aortic stenosis in high-risk patients using the second- and current third-generation self-expanding CoreValve prosthesis: device success and 30-day clinical outcome. J Am Coll Cardiol 2007; 50: 69-76.

5. Webb JG, Pasupati S, Humphries K. Percutaneous transarterial aortic valve replacement in selected high-risk patients with aortic stenosis. Circulation 2007; 116: 755-763.

6. Marcheix B, Lamarche Y, Berry C, et al. Surgical aspects of endovascular retrograde implantation of the aortic CoreValve bioprosthesis in high-risk older patients with severe symptomatic aortic stenosis. J Thorac Cardiovasc Surg 2007; 134: 1150-1156.

7. Webb JG, Altwegg L, Boone RH, et al. Transcatheter aortic valve implantation: impact on clinical and valve-related outcomes. Circulation 2009; 119: 3009-3016.

8. Grube E, Buellesfeld L, Mueller R, et al. Progress and current status of percutaneous aortic valve replacement: results of three device generations of the CoreValve Revalving system. Circ Cardiovasc Interv 2008; 1: 167-175. 